\title{
Giant drag reduction on Leidenfrost spheres evaluated from extended free-fall trajectories
}

\author{
Aditya Jetly, ${ }^{1}$ Ivan U. Vakarelski, ${ }^{1 *}$ Ziqiang Yang ${ }^{1}$ and Sigurdur T. Thoroddsen ${ }^{1}$ \\ ${ }^{1}$ Division of Physical Sciences and Engineering, King Abdullah University of Science and \\ Technology (KAUST), Thuwal 23955-6900, Saudi Arabia.
}

*ivanuriev.vakarelski@kaust.edu.sa

Tel: +966-12-8082119 


\section{ABSTRACT}

Vapor layer sustained on the surface of a heated sphere, by the means of the Leidenfrost effect, can dramatically reduce the hydrodynamic drag on the sphere due to an early drag crisis transition. Here we investigate the vapor layer effect on the free fall of heated metallic spheres in a fluorocarbon liquid, FC-72 (perfluorohexane), employing two tall liquid tanks: a 3 meter tall $14 \mathrm{~cm}$ wide tank and a 2 meter tall $20 \times 20 \mathrm{~cm}$ cross-section tank and heater device. These tanks are significantly larger than the tanks used in prior studies. We use highspeed video camera recordings to track extended fall trajectories and to compare the drag on room-temperature no-vapor-layer spheres to that of heated Leidenfrost vapor-layer spheres. Analysis of the extended free-fall trajectories and acceleration based on the sphere dynamic equation of motion enables the accurate evaluation of the vapor-layers-induced drag reduction, without the need for extrapolation. We demonstrate that the drag on the Leidenfrost sphere in FC-72, can be as low as $C_{\mathrm{D}}=0.04 \pm 0.01$, or an order of magnitude lower than the values for the no vapor layer spheres in the subcritical Reynolds numbers

range. This drag reduction extends into the supercritical Reynolds number range. The analysis method developed herein to describe the sphere trajectories can be applied in other related studies. Results of this study are expected to stimulate the development on energy saving drag reduction technologies based on lubricating gas layers.

Keywords: Drag reduction; Vapor layer; Leidenfrost effect; Drag crisis 


\section{Introduction}

Drag reduction by vapor or gas layers attached to solid surfaces in contact within a liquid is a topic of intense current research interest, which has been driven by the quest for the development of efficient energy saving technologies. The introduction of a gas layer on a solid moving in liquid is also an intriguing fluid dynamics phenomenon, as even a thin gas layer can modify the no-slip boundary condition at the solid surface and alter the flow patterns around the body and the related drag forces. Methods to induce gas layers at the solid body interface include bubbles injection, supercavitation, assisted cavitation [1], cavity entrapment during impact [2], and the use of superhydrophobic surfaces that can naturally sustain a thin air layer under water [3-6].

Recently the use of the Leidenfrost effect [7-9] has been shown to be a very efficient method to study gas layer drag reduction by sustaining a continuous vapor layer on the surface of solids which are heated to temperatures well above the boiling temperature of the surrounding liquid [10-14]. Initial experiments with heated Leidenfrost spheres falling in liquid were performed using a perfluorocarbon liquid, FC-72 mostly composed of perfluorohexane $\left(\mathrm{C}_{6} \mathrm{~F}_{14}\right)$ [11]. The FC-72, was preferred to water because of its much lower vaporization heat capacity (about 30 times lower) and lower boiling point of about $56{ }^{0} \mathrm{C}$, which makes it significantly easier to sustain a continuous vapor layer on the surface of the free falling hot sphere compared to water. 

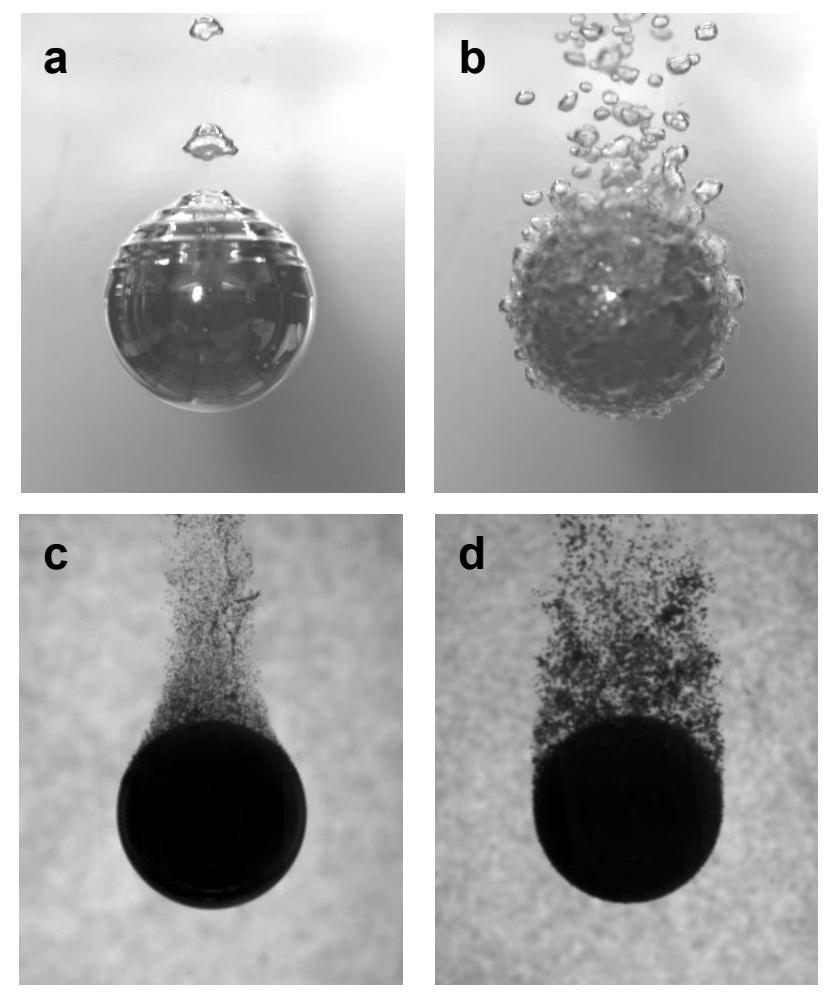

Fig. 1 (a, b) High-speed camera snapshots of a $20 \mathrm{~mm}$ steel sphere held stationary in FC -72: (a) when the sphere temperature, $T_{\mathrm{S}}$ is above the Leidenfrost temperature $T_{\mathrm{L}} \sim 110{ }^{\circ} \mathrm{C}$ and (b) shortly after passing the Leidenfrost temperature with explosive boiling. (c, d) High-speed camera snapshot silhouette images of a $20 \mathrm{~mm}$ steel sphere falling in FC-72: (c) for sphere temperature, $T_{\mathrm{S}} \sim 250{ }^{\circ} \mathrm{C}$ above the Leidenfrost temperature, and (d) at temperature slightly below the Leidenfrost temperature. See Vakarelski et al. 2011 [11] for related videos showing the entire process.

Fig. 1a shows a snapshot from a high-speed camera video illustrating the dynamic vapor layer streaming around a $20 \mathrm{~mm}$ diameter steel sphere held stationary in FC-72, when the sphere is heated well above the Leidenfrost temperature of about $110{ }^{\circ} \mathrm{C}$ and Fig. $1 \mathrm{~b}$ captures the moment of the vapor layer explosive destruction once the sphere temperature cools below the Leidenfrost transition temperature. By comparing the free fall velocities inside FC-72 of room temperature spheres with sphere heated above the Leidenfrost 
temperature it was demonstrated that the Leidenfrost vapor layer can reduce the hydrodynamic drag on sphere by up to $85 \%$ depending on the spheres Reynolds number [11]. The drag reduction is manifested as an early drag crisis transition, indicated by the wake separation point movement to the rear of the Leidenfrost spheres. Fig. 1c and Fig. 1d are snapshot silhouette images of a $20 \mathrm{~mm}$ steel sphere falling in FC-72, when the sphere temperature is above (Fig. 1c) or slightly below (Fig. 1d) the Leidenfrost temperature, demonstrating the delayed wake separation for the vapor-layer-shielded sphere.

In a later study [12] the Leidenfrost vapor layer effect was as well replicated for spheres falling in water. In this case a combination of superhydrophobic surface spheres and high water temperature, $T_{\mathrm{W}} \sim 85{ }^{\circ} \mathrm{C}$ to $95{ }^{0} \mathrm{C}$ was needed to sustain a stable vapor layer during the free falling. Similar approach was used in a modified Taylor-Couette experiment, which demonstrated that apart from the pressure component of the drag on falling sphere (form drag), the vapor layer can as well dramatically reduce the skin-friction component of the drag in turbulent flow conditions [13]. Most recently by using a range of high viscosity perfluorocarbon liquids it was shown that the Leidenfrost vapor layers drag reduction on falling sphere can occur for a very wide range of Reynolds number from as low as 600 up to $10^{5}$, far below the traditional drag crisis transition range [14]. Related numerical simulations using a Navier slip model with viscosity dependent slip length were shown to fit the experimental data [15].

In the initial investigation of the Leidenfrost vapor layers drag reduction on sphere free falling in FC-72, a 2 meter tall and $8 \mathrm{~cm}$ in diameters cylindrical liquid tank was used [11]. The size of the tank, which practically was determined by the amount of available perfluorocarbon liquid, thereby limited the size of the spheres and related Reynolds number range investigated. Here we re-examine the Leidenfrost vapor layer drag reduction in FC-72, by using two larger liquid tanks: a 3 meter tall and $14 \mathrm{~cm}$ in dimeter cylinder tanks, and a $2 \mathrm{~m}$ 
tall $20 \times 20 \mathrm{~cm}$ cross-section glass-windows tank and heater device (Fig. S1 in the supplementary material). Equally important is that in the present study we record and analyze the entire fall trajectory of the spheres, from the top to the bottom of the tank. In the prior study [11] we only measure the sphere falling velocity close to the bottom of the tank, assuming that it was close to the terminal velocity of the sphere, subsequently used to estimate the drag. Here, by tracking the fall velocity progression with time, we find that in fact most of the Leidenfrost spheres are still accelerating when reaching the tank bottom. In contrast, we now use the experimentally determined time dependences of the sphere velocity and acceleration to obtain an accurate evaluation of the drag reduction owning to the Leidenfrost vapor layer effect. We also use the capability of our liquid tank and heater device to conduct experiments with FC-72 fluid heated to temperature close to the boiling point.

\section{EXPERIMENTAL}

The fluid used (FC-72, 3M $\mathrm{M}^{\mathrm{TM}}$ Fluorinert ${ }^{\mathrm{TM}}$ Electronic Liquids) is a clear odorless, fully-fluorinated liquid, mostly composed of perfluorohexane $\left(\mathrm{C}_{6} \mathrm{~F}_{14}\right)$ with a boiling point of about $57{ }^{\circ} \mathrm{C}$, heat of vaporization, $H_{\mathrm{C}}=88 \mathrm{~kJ} / \mathrm{kg}$, and liquid density $\rho=1680 \mathrm{~kg} / \mathrm{m}^{3}$. Using an Ubbelohde type capillary viscometer (Fungilab) we measured the FC-72 dynamic viscosity at room temperature of about $21{ }^{\circ} \mathrm{C}$ to be $\mu=0.74 \pm 0.01 \mathrm{mPa}$ (slightly higher than the producer given value of $0.64 \mathrm{mPa} \mathrm{s}$ ) and at $55{ }^{\circ} \mathrm{C}$ to be $\mu=0.39 \pm 0.01 \mathrm{mPa}$.

The spheres used here were polished stainless steel spheres, $\rho_{\mathrm{s}}=7.8 \mathrm{~g} / \mathrm{cm}^{3}$, of diameters, $2 R=10$ to $60 \mathrm{~mm}$, or polished tungsten carbide spheres, $\rho_{\mathrm{s}}=14.8 \mathrm{~g} / \mathrm{cm}^{3}$, of diameters, $2 R=10$ to $45 \mathrm{~mm}$ purchased from various producers: FRITSCH GmbH, Zhuzhou Goode Tungsten Carbide Co., Ltd, and Simply Bearings, Ltd. Each sphere precise size was determined with a digital calibre and precise weight with a microbalance. 
Experiments were conducted in two liquid tanks. The first is a 3 meter tall and $14 \mathrm{~cm}$ in diameter cylindrical tank made of clear acryl (50 litres tank). The second tank is 2 meter tall, and has a cross-section area of $20 \times 20 \mathrm{~cm},(80$ litres tank) with front and back walls of double-glazed glass windows. An electric heater was installed at the bottom of this tank [12] that allowed the FC-72 liquid to be heated to $55{ }^{\circ} \mathrm{C}$ close to the boiling point of about $57{ }^{\circ} \mathrm{C}$. Fig. S1 in the supplementary material is a photograph of the two liquid tanks, where for comparison we also show the $2 \mathrm{~m}$ tall, $8 \mathrm{~cm}$ in dimeter clear acrylic tank (10 litres tank) used in our first investigation [11]. As the practical limiting factor for the tanks size is the available amount of FC-72 fluid, we chose to use taller, but narrower tanks to track the fall of the smaller spheres (10 to $25 \mathrm{~mm}$ ), and a shorter but wider tank to track the fall of the larger spheres (30 to $60 \mathrm{~mm})$.

The free fall of spheres in the tank was recorded with a high speed camera (Photron SA-5) using typical filming rates of $1000-2000$ fps. The sphere location vs time was determined by image processing the videos using the camera software (Photron FASTCAM Viewer, PFV Ver.3262). The usual coordinate sampling time interval was $20 \mathrm{~ms}$. From this data we evaluated the time dependence of the sphere fall velocities and acceleration used to estimate the spheres drag coefficient. The sphere velocity was calculated form the sphere coordinate data by using a moving median value over $\Delta t=100 \mathrm{~ms}$ time interval as data smoothing function. The sphere acceleration was respectively calculated from the velocity data as moving median over the same time interval. Further details on the drag coefficient estimation using the sphere velocity and acceleration are discussed in the following section (see Fig. 5 for example for the sphere coordinate $v s$ time, and related drag $v s$ time evaluation).

In the Leidenfrost spheres experiments, before release in the tank, we heat the sphere to $T_{\mathrm{S}}=250{ }^{\circ} \mathrm{C}$ in a controlled temperature furnace for at least 30 minutes. Using forceps the 
heated sphere is moved to the tank and carefully released from just below the FC-72 liquid surface. Using sphere temperature vs. cooling time data for perfluorohexane [14] we estimated that in all cases the sphere temperature drops by less than $15^{\circ} \mathrm{C}$ during the fall in the tank, which guarantees that over the entire fall the sphere remains in the Leidenfrost regime. The Leidenfrost state was as well confirmed by visual observation of the sphere cooling at the bottom of the tank following the fall. To account for the tanks wall effects we used the velocity correction formula given by Newton (1678):

$U / U_{\infty}=\left[1-\left(D / D_{T}\right)^{2}\right]\left[1-0.5\left(D / D_{T}\right)^{2}\right]^{1 / 2}$

where, $U$ is the measured velocity and $U_{\infty}$ is the corrected velocity for infinite flow domain, $D=2 R$ is the sphere diameter and $D_{\mathrm{T}}$ is the diameter of a cylindrical tank. For the $a \times a$ square cross-section tank, the effective diameter $D_{\text {T }}$ in Eq. (1) is calculated by equating the areas of the circle and the square, i. e. $D_{T}=(2 / \sqrt{\pi}) a$.

\section{Results and discussions}

\subsection{Falling spheres velocities}

The major set of experiments conducted compare the fall of room temperature spheres in FC-72, $T_{\mathrm{S}}=21{ }^{\circ} \mathrm{C}$ to the fall of identical spheres which were heated before release to the Leidenfrost sphere temperature, $T_{\mathrm{S}}=250{ }^{\circ} \mathrm{C}$. In the prior FC-72 Leidenfrost drag reduction study [11] we established that the fall velocity of the sphere sharply increases once the sphere temperature is above about $140{ }^{\circ} \mathrm{C}$, which secure a stable vapor layer on the falling sphere, and stays almost constant for $T_{\mathrm{S}}$ between $140{ }^{\circ} \mathrm{C}$ and $280{ }^{\circ} \mathrm{C}$. Respectively in the present 
study we use a standard sphere pre-heat temperature, $T_{\mathrm{S}}=250{ }^{\circ} \mathrm{C}$, for all sphere investigated as representative of the Leidenfrost regime case. For simplicity we will refer to the room temperature spheres experiment, $T_{\mathrm{S}}=21{ }^{\circ} \mathrm{C}$ as the "no vapor sphere case", and to the experiments conducted with spheres heated to $T_{\mathrm{S}}=250{ }^{\circ} \mathrm{C}$ as the "Leidenfrost sphere case".

Experiments with smaller diameters steel (ST) and tungsten carbide (TC) spheres; $2 R$ $=10 \mathrm{~mm}$ to $25 \mathrm{~mm}$ were conducted in the taller, 3 meter tank. For spheres of larger diameters, it was challenging to produce a sphere fall in the 3 meter tall tank without the sphere hitting the tank side wall, before reaching the bottom due to the inherit deviations from the straight fall of the sphere at that range of Reynolds numbers [14]. Alternatively, the larger size spheres, ST of $2 R=30$ to $60 \mathrm{~mm}$ and TC of $2 R=30$ to $45 \mathrm{~mm}$, experiments were conducted using the wider, 2 meter tall $20 \times 20 \mathrm{~cm}$ cross-section tank in which it was possible to produce a free-fall without the sphere coming close to the tank walls.

Fig. 2 presents results for the velocity progression of selected sizes of the steel spheres, and Fig. 3 for tungsten carbide spheres falling in the 3 meter tall $(L=3 \mathrm{~m})$ or the 2 meter tall $(L=2 \mathrm{~m})$ tanks. Each Fig. panel compares the velocity of identical spheres for room temperature (no vapor layer) case sphere and the heated Leidenfrost spheres. Results for other sphere sizes are given as Fig. S2 in the supplementary material for the ST spheres and Fig. S3 in the supplementary material for the TC spheres.

The data in Fig. 2 and Fig. 3 demonstrate the magnitude of the Leidenfrost sphere velocity increase due to the vapor layer drag reduction effect over the range of ST and TC sphere sizes investigated. The dramatic difference in the fall velocities between the no vapor layer and Leidenfrost vapor layer lubricated spheres is best demonstrated by the Supplementary material Video 1 which shows $20 \mathrm{~mm}$ steel spheres fall toward the lower part of the 3 meter tall tank. More importantly the Fig. 2 and Fig. 3 show that except for the smallest $10 \mathrm{~mm}$ steel sphere studied here, all other Leidenfrost spheres are still accelerating 
when reaching the bottom of the tank, even though in this study we use taller and wider tanks than in our previous study. For most of sphere sizes investigated the no vapor layer spheres reach terminal velocity before hitting the bottom of the tank. However, the largest sphere sizes studied (Fig. 2d and Fig. 3d) are also accelerating when reaching the tank bottom, indicating a transition to drag crisis regime in the no vapor case. The transition to the drag crisis regime during the free-fall is indicated by the sudden change in the slope of the velocity curves, as is readily noticeable for the $2 R=15 \mathrm{~mm}$ Leidenfrost sphere in Fig. $2 \mathrm{~b}$ and for the $2 R=10 \mathrm{~mm}$ TC Leidenfrost case in Fig. 3a.
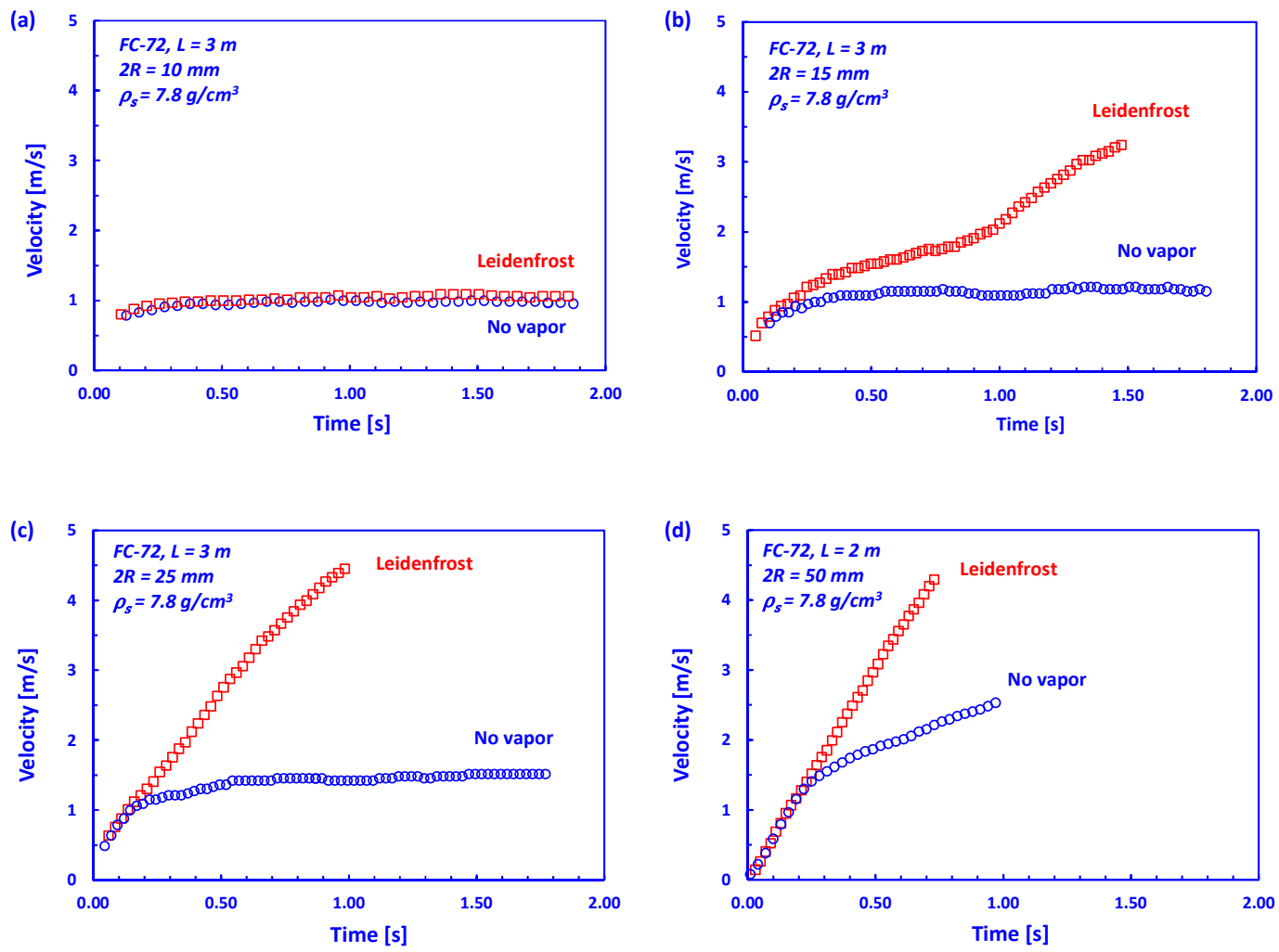

Fig. 2 Comparison of spheres fall velocity progression with time, during the fall of identical steel spheres in FC-72, of room temperature, $T_{\mathrm{S}}=21{ }^{0} \mathrm{C}$ (blue circles) or under Leidenfrost regime, $T_{\mathrm{S}}=250{ }^{\circ} \mathrm{C}$ (red squares): (a) $2 R=10 \mathrm{~mm}$, (b) $15 \mathrm{~mm}$, (c) $25 \mathrm{~mm}$, and (d) $2 R=50$. See Fig. S2 in the supplementary material for the other steel sphere sizes. 

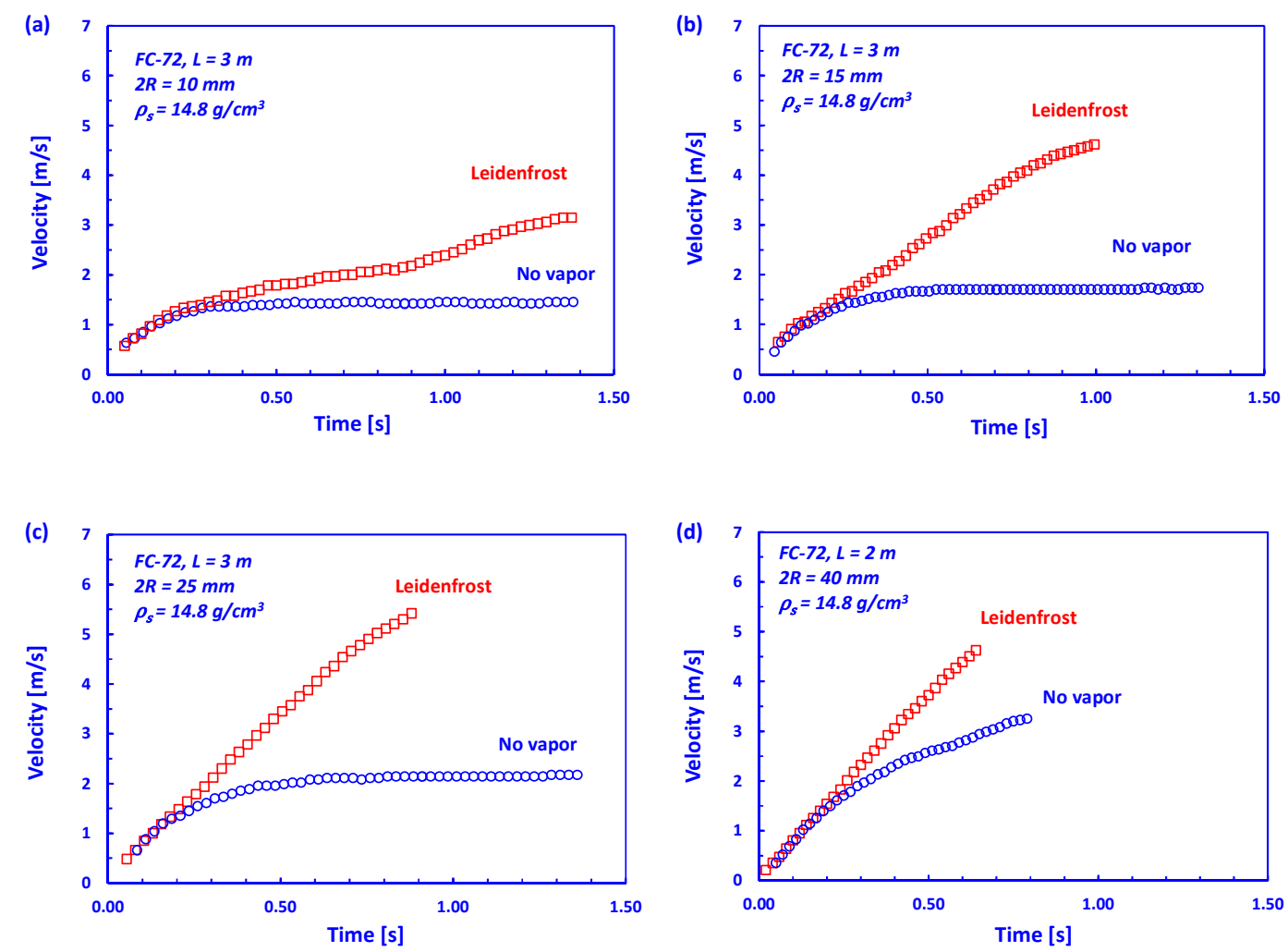

Fig. 3 Comparison of spheres fall velocity progression with time during the fall of identical tungsten carbide spheres in FC-72, for sphere of room temperature, $T_{\mathrm{S}}=21{ }^{\circ} \mathrm{C}$ (blue circles) or Leidenfrost spheres, $T_{\mathrm{S}}=250{ }^{\circ} \mathrm{C}$ (red squares): (a) $2 R=10 \mathrm{~mm}$, (b) $15 \mathrm{~mm}$, (c) $25 \mathrm{~mm}$ and (d) $2 R=40 \mathrm{~mm}$. See Fig. S3 in the supplementary material for the other tungsten carbide sphere sizes.

An additional set of experiments was conducted in the 2 meter tall tank and heater device using FC-72 liquid heated to $55{ }^{\circ} \mathrm{C}$. In this case we used a range of steel sphere pre-heated to the liquid temperature, $T_{\mathrm{S}}=55{ }^{\circ} \mathrm{C}$ to the fall of identical steel spheres which were heated before release to the Leidenfrost sphere temperature, $T_{\mathrm{S}}=160{ }^{0} \mathrm{C}$. The velocity progressions with time data for these experiments are presented in the supplementary materials Fig. S4, and related drag coefficient calculated from these data will be discussed below. 


\subsection{Falling sphere equation of motion}

Here we review the dynamical equation of the spheres motion during the free fall to derive some simplified expressions for the relation between the sphere velocity, acceleration and drag. As usually the drag on the sphere is characterized, as drag coefficient, $C_{\mathrm{D}}=$ $2 F_{\mathrm{D}} /\left(\pi R^{2} \rho U^{2}\right)$, as function of the Reynolds number, $\operatorname{Re}=2 \rho R U / \mu$. Here $F_{\mathrm{D}}$ is the drag force on the sphere, $\rho$ the fluid density, $R$ the sphere radius and $\mu$ the dynamic viscosity of the fluid.

The dynamic equation of motion of the sphere, during the free fall in still Newtonian fluids is given by the Basset-Boussinesq-Oseen (BBO) equation, which for the Reynolds number range in our experiment can be written as [16-18]:

$m_{S} \frac{d U}{d t}=\left(m_{S}-m\right) g-\frac{C_{D}}{2} \rho\left(\frac{\pi D^{2}}{4}\right) U^{2}-k m_{S} \frac{d U}{d t}-\frac{3}{2} D^{2} \sqrt{\pi \rho \mu} \int_{0}^{t} \frac{d U / d \tau}{\sqrt{t-\tau}} d \tau$

where $g$ is the acceleration of the gravity, $D=2 R, m_{\mathrm{s}}$ is the sphere mass, $m$ the mass of the displaced fluid, $k$ is the added mass coefficient (for subcritical Re spheres, $k \approx 0.5$ ). The left hand side of the equation is the sphere inertia force. The right-hand side first, second, third and fourth terms are respectively the submerged weight, drag, added mass and the Basset history force. If the dependence of the drag coefficient on the velocity is known for the entire fall, this equation can be solved numerically to obtain the sphere fall trajectory and velocity time dependencies [16]. For the high Reynolds number conditions of our experiment and relevantly high density of the spheres compared to that of the surrounding fluid, the Basset history force has been shown to be negligible (less than 1\%) [16] and can be therefore omitted to a good approximation, to simplify Eq. (2) to: 
$\left(\rho_{S}+k \rho\right) \frac{d U}{d t}=\left(\rho_{S}-\rho\right) g-\frac{3 \rho C_{D}}{4 D} U^{2}$

where we have now expressed the sphere and displaced fluid masses using the sphere and fluid densities, $\rho_{\mathrm{S}}$ and $\rho$. For a constant drag coefficient $C_{\mathrm{D}}$, this equation can be solved analytically by applying the method of the partial fractions. Setting the initial condition as $U$ $=0$ at $t=0$ we find the solution:

$U=U_{T} \tanh \left(\frac{t}{\tau}\right)=U_{T}\left(\frac{1-e^{-2 t / \tau}}{1+e^{-2 t / \tau}}\right)$

where:

$U_{T}=\left(\frac{4 D}{3 \rho C_{D}}\left(\rho_{S}-\rho\right) g\right)^{1 / 2}$

and

$$
1 / \tau=\frac{\left(\frac{3 \rho C_{D}}{4 D}\left(\rho_{S}-\rho\right) g\right)^{1 / 2}}{\left(\rho_{S}+k \rho\right)} .
$$

More generally this solution holds for any segment of the sphere fall during which the drag coefficient is constant, by offsetting the time at the beginning of the segment to match the velocity value, $U_{0}$ at $t=t_{0}$ :

$U=U_{T} \tanh \left(\frac{t-\left(t_{0}-t_{0}^{1}\right)}{\tau}\right), \quad$ for $t>t_{0}$ and $C_{\mathrm{D}}=$ constant

where $t_{0}{ }^{1}$ satisfies:

$U_{0}=U_{T} \tanh \left(\frac{t_{0}^{1}}{\tau}\right)$ 
It is well known that in the subcritical Reynolds number regime, $\operatorname{Re}=10^{3}$ to $2 \times 10^{5}$ the drag coefficient has a plateau value of about 0.45 to 0.55 . We have further noticed that for the range of ST and TC spheres sizes used in our experiment, the velocities corresponding to $\operatorname{Re}>10^{3}$ is less than $1 / 10$ of the spheres terminal velocities, and is reached shortly after the sphere is released in the tank. Respectively, in Fig. 4 we demonstrate that for the no vapor spheres falling at subcritical Reynolds numbers, the $U$ vs. $t$ experimental dependences closely follow the Eq. (4) given solution. The Fig. 4 example is for the $2 R=25 \mathrm{~mm} \mathrm{ST}$ and $2 R=25$ $\mathrm{mm}$ TC no vapor spheres falling in FC-72 and Fig. $4 \mathrm{~b}$ for is the same spheres falling in room temperature water. In the case of Fig. $4 \mathrm{~b}$ we used data collected in our previous study [6] for sphere falling in a water-filled tank of 2.5 meter height and $40 \times 40 \mathrm{~cm}$ cross-section, which results in less sideway velocity fluctuation due to the tank wall effects.

In some of the previous studies $[12,19,20]$ on drag on free-falling spheres the $U$ vs. $t$ data were extrapolated using the empirical exponential relation:

$U=U_{T}\left(1-e^{-t / \tau_{1}}\right)$

where $U_{\mathrm{T}}$ and $\tau_{1}$ are two independent fitting parameters. However, the examples in Fig. 4 show very good agreement with the Eq. (4) solution of the simplified BBO equation, using only one independent fitting parameter, which could be the drag coefficient, $C_{\mathrm{D}}$ that determines both the terminal velocity, $U_{\mathrm{T}}$ and the characteristic time $\tau$ according to Eq. (5) and Eq. (6) respectively. Alternatively, one can use the experimentally determined $U_{\mathrm{T}}$ value to calculate $C_{\mathrm{D}}$ and $\tau$. The two empirical parameters Eq. (9) can still be useful in some cases, as they were shown to be effective in predicting the terminal velocities of spheres including cases when the drag varies during the fall [19]. 

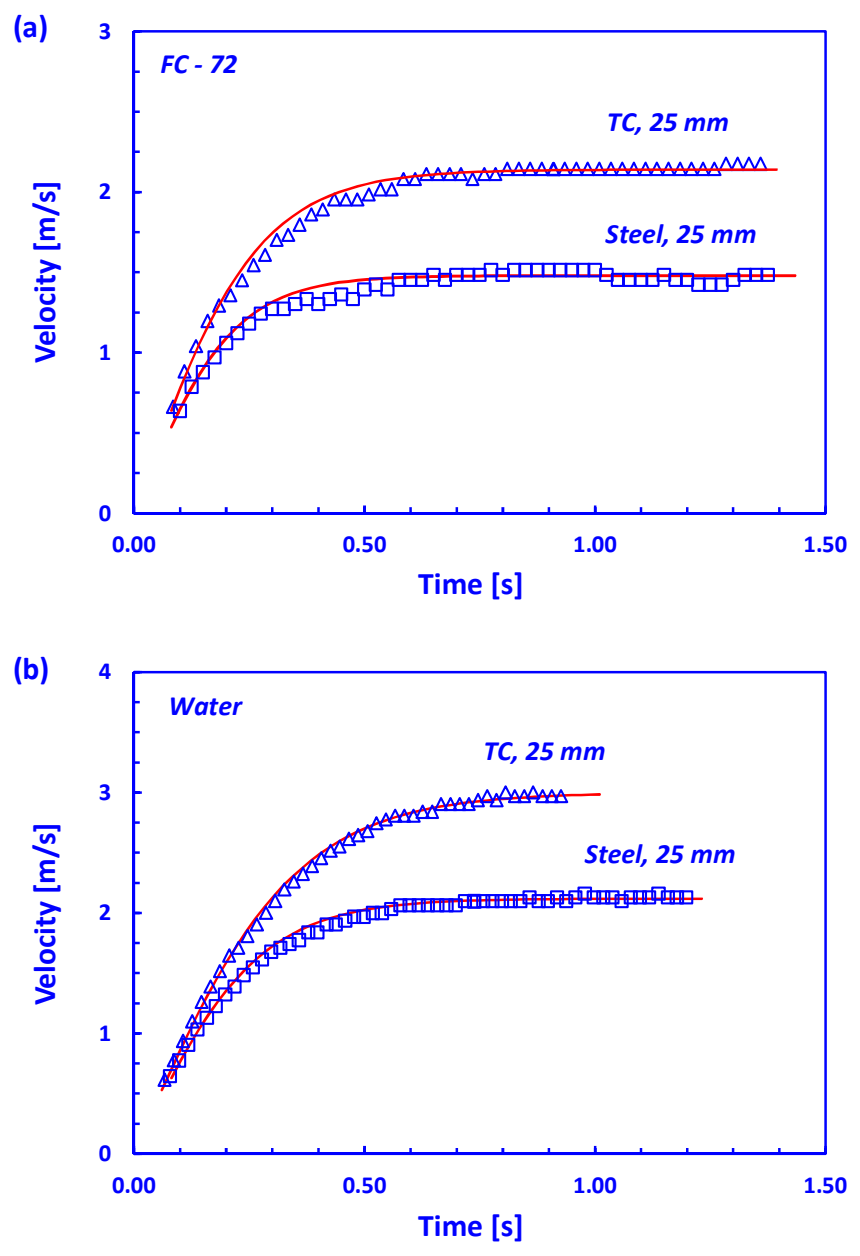

Fig. 4 Comparison of the experimental sphere free-fall velocity progression with time with the Eq. (4) analytical solution of the BBQ equation assuming a constant drag coefficient fall. (a) Fall of $2 R=25 \mathrm{~mm}$ steel sphere (squares) and $2 R=25 \mathrm{~mm}$ tungsten carbide (triangles) in FC-72 measured in the 3 meters tall tank. (b) Fall of the same spheres in water measured in a 2.5 meter tall tank (experimental data from Jetly et al. [6]). The red lines are Eq. (4) fits, where $C_{\mathrm{D}}$ and $\tau$ were determined from the experimental value of the terminal velocity, $U_{\mathrm{T}}$ using Eq. (5) and Eq. (6) respectively. 


\subsection{Evaluation of the drag coefficients}

The drag coefficient of spheres falling at terminal velocity is usually determined using the below relation which follows from the balance of buoyancy gravity, and drag force:

$C_{D}=\frac{4 D}{3 \rho U_{T}^{2}}\left(\rho_{S}-\rho\right) g$

which is indeed identical to Eq. (5). However, in most of the cases of Leidenfrost spheres considered herein and for the lager no vapor spheres (Fig. 2 and Fig. 3) the sphere is still accelerating reaching the bottom of the tank. In these cases Eq. (3) can be used to estimate the instantaneous value of the drag coefficient, by accounting for the sphere acceleration as:

$C_{D}=\frac{4 D}{3 \rho U^{2}}\left(\left(\rho_{S}-\rho\right) g-\left(\rho_{S}+0.5 \rho\right) \frac{d U}{d t}\right)$

This equation converges to Eq. (10) for spheres falling at terminal velocity, e.g. for which

$\frac{d U}{d t}=0$. Here we remind that in Eq. (3) and respectively Eq. (11) we ignore the history force which is expected to be negligible in our experiments, and we use the approximate value of the added mass coefficient, $k=0.5$. The standard added mass coefficient, $k=0.5$ value for a sphere has been experimentally verified for Re up to about $2.5 \times 10^{5}[22,23]$. To the authors knowledge there is no well-established added mass coefficient correction at supercritical Reynolds number [18]. However as in our system the sphere density is substantially higher than the liquid density (4 to 8 times) the drag coefficient estimate is not very sensitive to possible deviation from the $k=0.5$ value. 
Fig. 5 shows an example for the evaluation of the instantaneous drag coefficient using Eq. (11). Fig. 5a shows the sphere depth vs. time data, $x(t)$, for the $2 R=25 \mathrm{~mm} \mathrm{ST}$ and $2 R=$ $25 \mathrm{~mm}$ TC Leidenfrost spheres falling in the $3 \mathrm{~m}$ tank. Fig. $5 \mathrm{~b}$ is the corresponding velocity $v s$ time dependence, $U(t)=\mathrm{d} x(\mathrm{t}) / \mathrm{d} t$, calculated as moving median from the $x(t)$ data. Fig. 5c is the corresponding drag coefficient $v s$ time calculated using Eq. (11) in which the acceleration, $\mathrm{d} U(\mathrm{t}) / \mathrm{d} t$ is calculated as moving median from the $U(\mathrm{t})$ data. The most significant result illustrated in Fig. 5 is that, while the sphere velocity is far from the terminal value at the end of the fall (Fig. 5b), the drag coefficient on the sphere reaches a plateau value (Fig. $5 \mathrm{c}$ ), at a time corresponding to a depth of about 0.5 meters. This result is important from practical point of view as we can now evaluate the drag coefficients using tanks that are substantially shorter than the one that will be needed for the spheres to reach a velocity close to the terminal velocity value.

Next we convert the drag coefficient $v s$ time data to the corresponding dependences on the spheres instantaneous Reynolds number. In Fig 6 we show such $C_{\mathrm{D}}$ vs Re dependences evaluated from the fall trajectories of selected sizes of steel and tungsten carbide Leidenfrost spheres. For all spheres shown the drag coefficient in the beginning of the fall is close to the drag of the no-vapor-layer sphere, starting from the values in subcritical regime, i. e. $C_{\mathrm{D}} \sim$ $0.5-0.6$. We assume that it takes some time from the start of the free fall for the flow separation around the Leidenfrost sphere to settle to the rear of the sphere, corresponding to the low-drag-value plateau. It seems that the plateau values dependence on the Reynolds number approaches a universal dependence for the range of sphere sizes and densities investigated. 

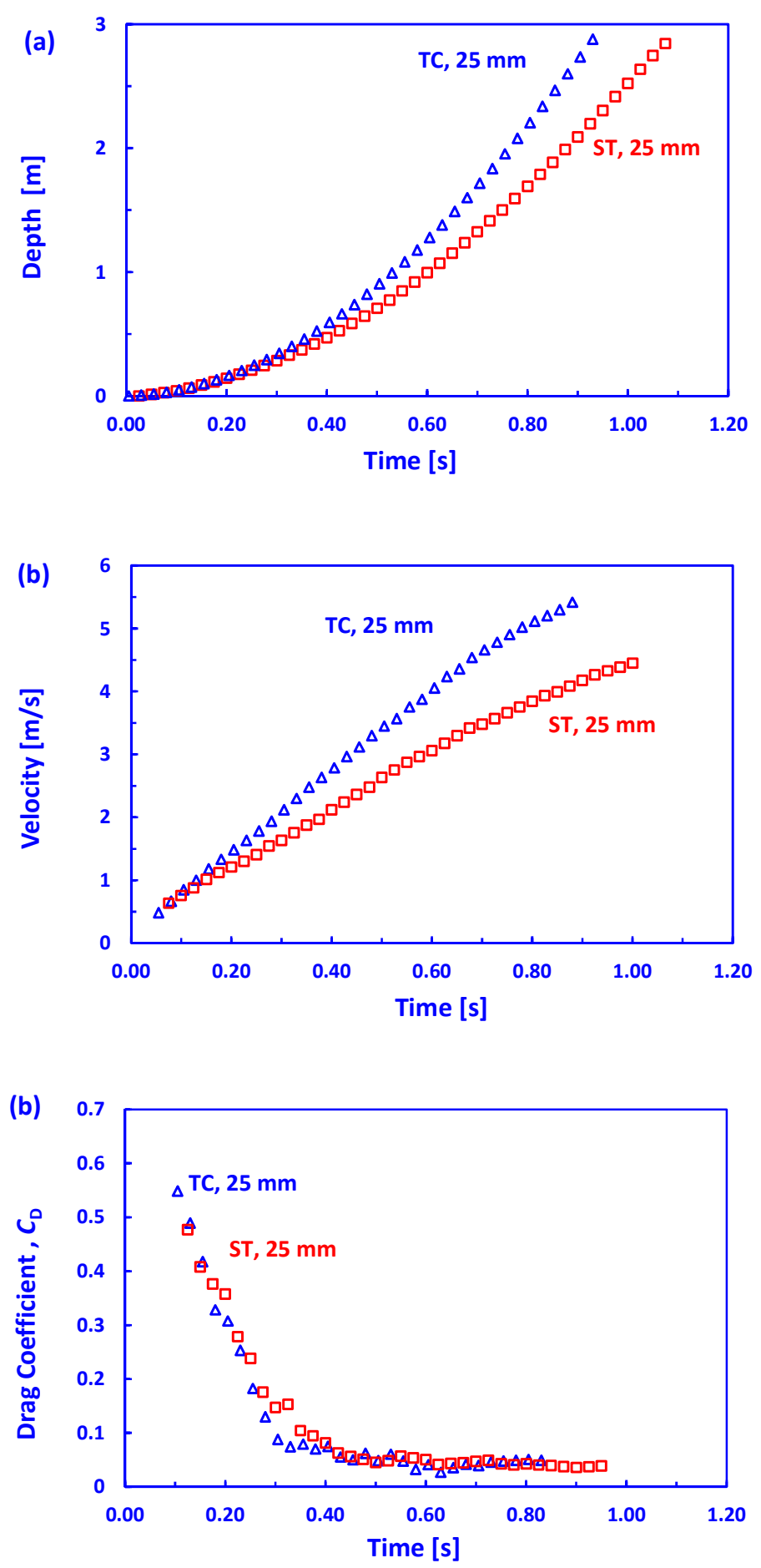

Fig. 5 Procedure to calculate $C_{\mathrm{D}}$. Sphere depth coordinate (a), the corresponding fall velocity (b) and drag coefficient (c) vs. time during the fall of a $2 R=25 \mathrm{~mm}$ steel Leidenfrost sphere (red squares) and $2 R=25 \mathrm{~mm}$ tungsten carbide Leidenfrost sphere (blue triangles) in FC-72 
measured in the 3 meters tall tank. (a) The spheres depth coordinate, $x(t)$ is measured from the high-speed videos using a $25 \mathrm{~ms}$ sampling rate. (b) The sphere velocity is calculated as moving median time differential of the coordinate over a time interval, $\Delta t=125 \mathrm{~ms}$, using data smoothing function, e. g. $U(t)=(x(t+0.5 \Delta t)-x(t-0.5 \Delta t)) / \Delta t$. (c) The drag on the sphere calculated using Eq. (11), where the sphere acceleration, $\mathrm{d} U / \mathrm{d} t$ was calculated as moving median of the velocity time differential, over $\Delta t=125 \mathrm{~ms}$.

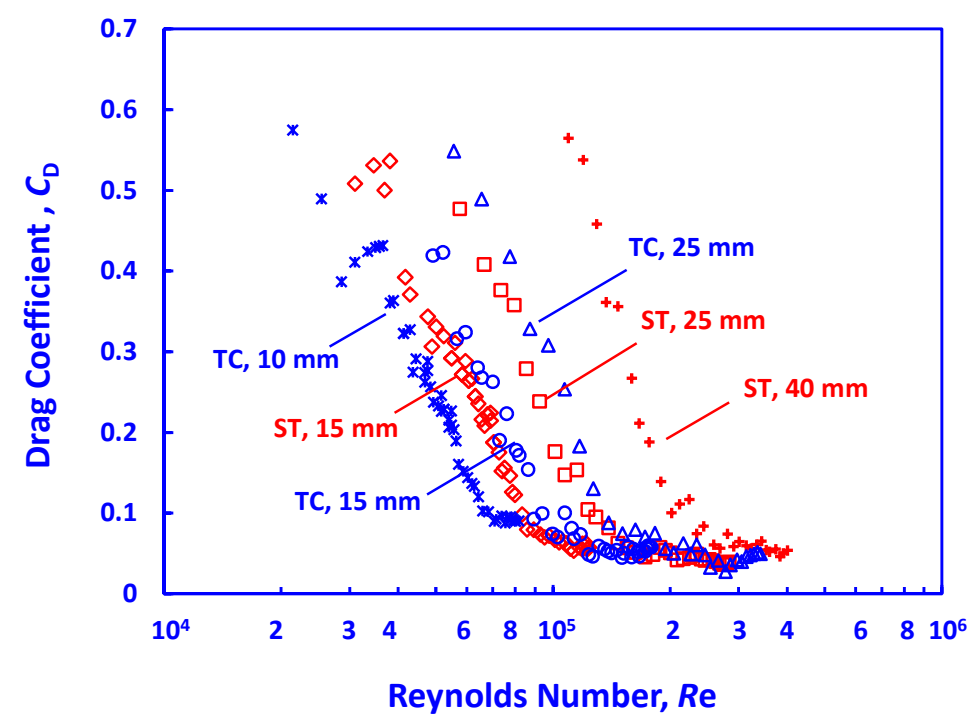

Fig. 6 Drag coefficient vs Reynold number for selected steel (ST, 2R $=15,25,40 \mathrm{~mm}$ ) Leidenfrost spheres and tungsten carbide (TC, $2 R=10,15,25 \mathrm{~mm})$ Leidenfrost spheres. Each data set is evaluated from the sphere fall trajectory data, as done in the Fig. 5, and plotted vs. the sphere instantaneous Reynolds number. 
Finally in Fig. 7 we present the $C_{\mathrm{D}}$ dependence on the Reynolds number, calculated using all studied steel and tungsten carbide spheres fall trajectories data in the no vapor and Leidenfrost cases. Each data point is calculated using Eq. (11), where the sphere velocity, $U(\mathrm{t})$ and acceleration $\mathrm{d} U(\mathrm{t}) / \mathrm{d} t$ values are measured close to the bottom of the 3 meter or the 2 meter tank. Fig. 7a uses a linear vertical axis for the drag coefficient data and Fig. 7b shows the same data using a logarithmic vertical axis for the drag coefficient values to more clearly present the spread in the Leidenfrost spheres drag coefficient data.

The $C_{\mathrm{D}}$ data for the no vapor cases closely follow the literature values, with subcritical range of $C_{\mathrm{D}} \sim 0.45-0.55$ followed by drag crisis transition at $\mathrm{Re} \sim 2.5$ to $3.5 \times$ $10^{5}$ and supercritical values, $C_{\mathrm{D}} \sim 0.15-0.20[12,19,23,24]$. Notice the good agreement between the literature drag coefficient values calculated from the terminal velocity of the sphere falling in a very large $(\sim 6.5$ meter tall) tank [23] and our 2 meter tank data obtained using Eq. (11).

As can be expected the results for the Leidenfrost sphere follow the trend outlined in our prior study of an early drag crisis transition starting from $\operatorname{Re} \sim 4 \times 10^{4}$. However, here by using a larger tank and accounting for the sphere acceleration we measure drag coefficient values, $C_{\mathrm{D}} \sim 0.04 \pm 0.01$, which are about twice lower than those measured in the smaller tank. We as well show, that the vapor-layer drag reduction effect persists for supercritical Reynolds numbers, $\operatorname{Re}>3.5 \times 10^{5}$. Thus, the vapor layer reduces the drag by an order of magnitude in the subcritical Reynolds number range, and several fold for the supercritical Reynold number range investigated here (Fig. 7b). We note that similar range of drag reduction at supercritical Reynolds number was hinted for Leidenfrost spheres falling in heated $95{ }^{\circ} \mathrm{C}$ water [12]. However in this case the estimation was based on drag coefficients calculated using extrapolated values of the sphere fall velocities whereas in the present study the drag coefficients are calculated using the measured sphere velocity and acceleration. 

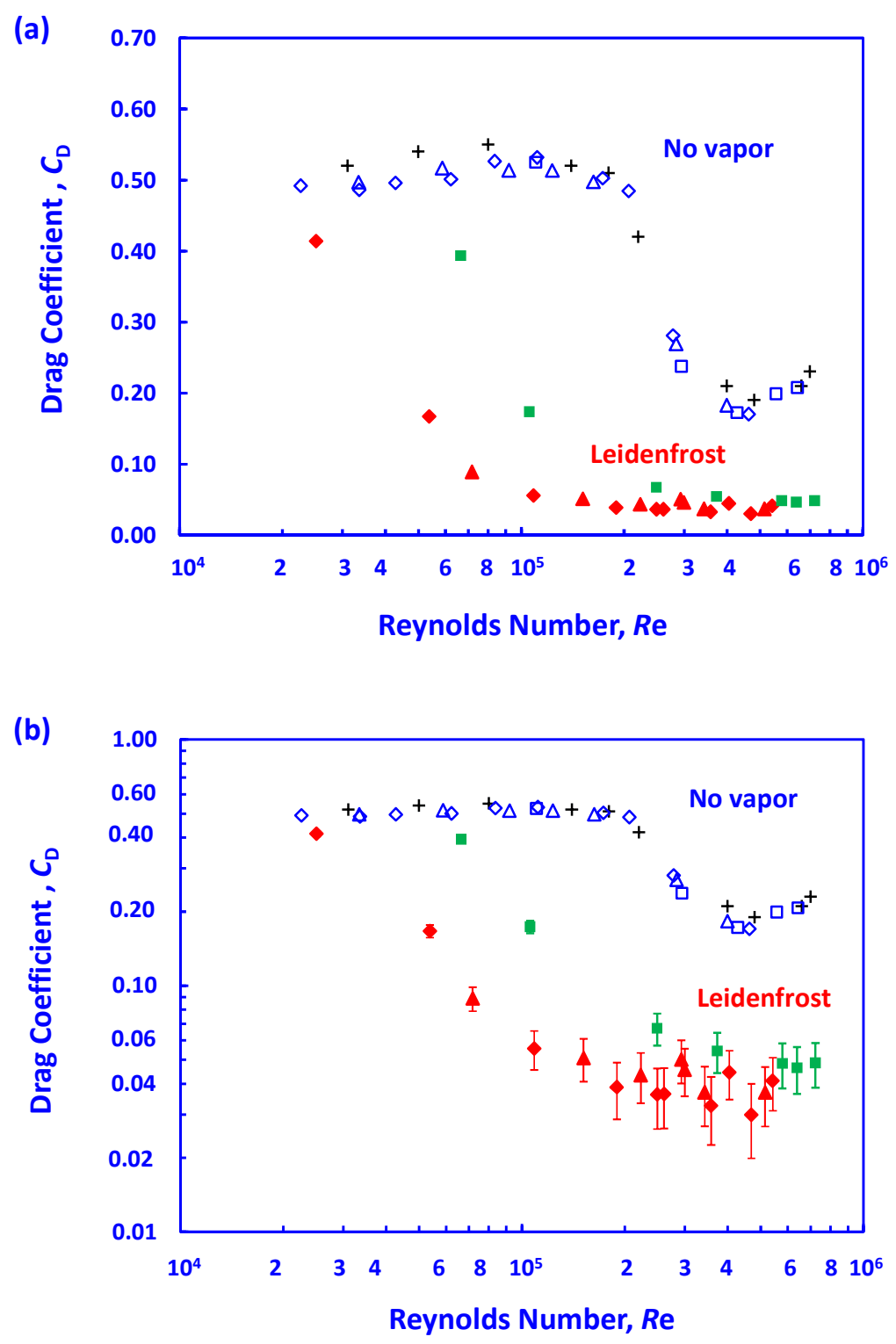

Fig. 7 Drag coefficient, $C_{\mathrm{D}}$ vs Reynold number, $R e$ dependence for the room temperature no vapor spheres, $T_{\mathrm{S}}=21{ }^{\circ} \mathrm{C}$ and Leidenfrost sphere, $T_{\mathrm{S}}=250{ }^{\circ} \mathrm{C}$ falling in FC-72. Panel (a) uses a linear vertical axis and (b) uses a logarithmic vertical axis for the $C_{\mathrm{D}}$ values. Each data point is calculated using Eq. (11) with the sphere velocity and acceleration taken close to the end of the $3 \mathrm{~m}$ or $2 \mathrm{~m}$ tank fall. Open diamonds (blue) are for the no-vapor steel spheres, open triangles (blue) for the no-vapor tungsten carbide sphere. Solid diamonds (red) are for Leidenfrost steel spheres and solid triangles (red) for Leidenfrost tungsten carbide spheres. 
Solid squares (green) are data for Leidenfrost steel sphere falling in FC-72 heated to $55{ }^{\circ} \mathrm{C}$. Literature values for no vapor steel spheres falling in an "infinite" water tank measured by White [23] are shown as crosses.

To the authors knowledge the values of the drag coefficients on the Leidenfrost spheres, $C_{\mathrm{D}} \sim 0.04$ measured here are the lowest demonstrated for a free falling sphere. The vapor layer drag reduction compares favourably to any alternative drag reduction approach that induces an early drag crisis, as the use of surface modification with roughness feature [25], addition of flexible polymers $[16,19,23]$ or mass transfer [20]. We note however that the Leidenfrost data shown in Fig. 7 are for the specific case of heated metallic spheres falling in room temperature FC-72. The magnitude of the drag reduction by the gas layer will depend on the properties of the gas layer, most importantly the gas layer thickness and the ratio between the fluid and gas viscosities. This is demonstrate in Fig. 7 by the data collected for FC-72 heated to the $55{ }^{\circ} \mathrm{C}$, at which temperature FC-72 has a lower viscosity then the room temperature FC-72, resulting in a shift to higher Reynolds numbers of the drag reduction transition. Respectively for high viscosity liquids the drag reduction effect is shifted to lower Reynolds numbers [14]. At the other hand the thinner but practically relevant air layers sustained on superhydrophobic surface sphere falling in water produces a more significant drag reduction at Reynolds numbers that are closer to the critical Reynolds number of the no-vapor spheres [6].

\section{Conclusion}

Here we investigated the drag reduction induced by Leidenfrost vapor layer sustained on the surface of heated metallic spheres falling in the perfluorocarbon liquid, FC-72, using 
liquid tanks which are substantially larger than the one used in prior studies. Nevertheless we found that for most of the cases of Leidenfrost sphere investigated, the spheres were still accelerating when they reach the tank bottom. Analyzing the equation of the sphere motion we derive simplified expressions that correctly predicted the sphere fall trajectory in the case of known drag coefficient. Respectively we used these equations to estimate the sphere drag coefficient accounting for the experimental measured sphere velocity and acceleration progression. A practically important result from this analysis is that for the range of Leidenfrost sphere investigated, during the fall the drag coefficient reaches a plateau value, well before the sphere reaches terminal velocity. The methodology for analyzing the free-fall trajectories demonstrated here can be used in any related study of free-falling spheres. The drag coefficients value for the Leidenfrost sphere of about 0.04 are arguably the lowest $C_{\mathrm{D}}$ measured for a free falling sphere and the closest practical realization of a non-slip sphere falling in the super-critical Reynolds numbers range.

\section{REFERENCES}

[1] S.L. Ceccio, Friction drag reduction of external flows with bubble and gas injection, Annu. Rev. Fluid Mech. 42 (2010) 183-203. https://doi.org/10.1146/annurev-fluid$\underline{121108-145504}$

[2] I.U. Vakarelski, E. Klasboer, A. Jetly, M.M. Mansoor, A.A. Aguirre-Pablo, D.Y.C. Chan, S.T. Thoroddsen, Self-determined shapes and velocities of giant near-zero drag gas cavities, Sci. Adv. 3 (2017) e1701558. https://doi.org/10.1126/sciadv.1701558

[3] J.P. Rothstein, Slip on superhydrophobic surfaces, Annu. Rev. Fluid Mech. 42 (2010) 89-109. https://doi.org/10.1146/annurev-fluid-121108-145558 
[4] G. McHale, M.R. Flynn, M.I. Newton, Plastron induced drag reduction and increased slip on a superhydrophobic sphere, Soft Matter 7 (2011) 10100-10107. https://doi.org/10.1039/c1sm06140b

[5] H. Dong, M. Cheng, Y. Zhang, H. Wei, F. Shi, Extraordinary drag-reducing effect of a superhydrophobic coating on a macroscopic model ship at high speed. J Mater Chem A 1 (2013) 5886-5891. https://doi.org /10.1039/c3ta10225d

[6] A. Jetly, I.U. Vakarelski, S.T. Thoroddsen, Drag crisis moderation by thin air layers sustained on superhydrophobic spheres falling in water, Soft Matter 14 (2018) 16081613. https://doi.org/10.1039/c7sm01904a

[7] J.G. Leidenfrost, "De Aquae Communis Nonnullis Qualitatibus Tractatus,” (1756); J. G. Leidenfrost, Int. J. Heat Mass Transfer 9 (1966) 1153-1166. https://doi.org/10.13140/RG.2.1.1442.7045

[8] I.U. Vakarelski, N.A. Patankar, J.O. Marston, D.Y.C. Chan, S.T. Thoroddsen, Stabilization of Leidenfrost vapour layer by textured superhydrophobic surfaces, Nature 489 (2012) 274-277. https://doi.org/10.1038/nature11418

[9] D. Quéré, Leidenfrost dynamics, Annu. Rev. Fluid Mech. 45 (2013) 197-215. https://doi.org/10.1146/annurev-fluid-011212-140709

[10] W. Bradfield, R. Barkdoll, J. Byrne, Some effects of boiling on hydrodynamic drag, Int. J. Heat Mass Transf. 5 (1962) 615-620. https://doi.org/10.1016/0017-9310(62)90085-6

[11] I.U. Vakarelski, J.O. Marston, D.Y.C. Chan, S.T. Thoroddsen, Drag reduction by Leidenfrost vapor layers, Phys. Rev. Lett. 106 (2011) 214501. https://doi.org/10.1103/PhysRevLett.106.214501 
[12] I.U. Vakarelski, D.Y.C. Chan, S.T. Thoroddsen, Leidenfrost vapour layer moderation of the drag crisis and trajectories of superhydrophobic and hydrophilic spheres falling in water, Soft Matter 10 (2014) 5662-5568. https://doi.org/10.1039/c4sm00368c

[13] D. Saranadhi, D. Chen, J.A. Kleingartner, S. Srinivasan, R.E. Cohen, G.H. McKinley, Sustained drag reduction in a turbulent flow using a low-temperature Leidenfrost surface, Sci. Adv. 2 (2016) e1600686. https://doi.org/10.1126/sciadv.1600686

[14] I.U. Vakarelski, J.D. Berry, D.Y.C. Chan, S.T. Thoroddsen, Leidenfrost vapor layers reduce drag without the crisis in high viscosity liquids, Phys. Rev. Lett. 117 (2016) 114503. https://doi.org/10.1103/PhysRevLett.117.114503

[15] J.D. Berry, I.U. Vakarelski, D.Y.C. Chan, S.T. Thoroddsen, Navier slip model of drag reduction by Leidenfrost vapor layers, Phys. Fluids 29 (2017) 107104. https://doi.org/10.1063/1.4993298

[16] K. Watanabe, H. Kui, I. Motosu, Drag of a sphere in dilute polymer solutions in high Reynolds number range, Rheol. Acta 37 (1998) 328-335.

https://doi.org/10.1007/s003970050119

[17] M.M. Mansoor, I.U. Vakarelski, J.O. Marston, T.T. Truscott, S.T. Thoroddsen, Stable-streamlined and helical cavities following the impact of Leidenfrost spheres, J. Fluid Mech. 823 (2017) 716-754. https://doi.org/10.1017/jfm.2017.337

[18] Z. Yin, Z. Wang, B. Liang, L. Zhang, Initial velocity effect on acceleration fall of a spherical particle through still fluid, Mathematical Problems in Engineering (2017) 9795286. https://doi.org/10.1155/2017/9795286

[19] N. Lyotard, W.L. Shew, L. Bocquet, J.-F. Pinton, Polymer and surface roughness effects on the drag crisis for falling spheres, Eur. Phys. J. B 60 (2017) 469-476. https://doi.org/10.1140/epjb/e2008-00018-0 
[20] W.S. Hamilton, J.E. Lindell, Fluid force analysis and accelerating sphere tests. Journal of the Hydraulics Division 97 (1971) 805-817.

[21] J. Pantaleone, J Messer, The added mass of a spherical projectile, American Journal of Physics 79 (201) 1202-1210. https://doi.org/10.1119/1.3644334

[22] I.U. Vakarelski, D.Y.C. Chan, S.T. Thoroddsen, Drag moderation by the melting of an ice surface in contact with water, Phys. Rev. Lett. 115 (2015) 044501. https://doi.org/10.1103/PhysRevLett.117.114503

[23] A. White, Drag on spheres in diluted high polymer solutions, Nature 216 (1967) 994995. https://doi.org/10.1038/216994b0

[24] E. Achenbach, Experiments on the flow past spheres at very high Reynolds numbers, J. Fluid Mech. 54 (1972) 565-575. https://doi.org/10.1017/S0022112072000874

[25] J. Choi, W.-P. Jeon, H. Choi, Mechanism of drag reduction by dimples on a sphere, Phys. Fluids 18 (2006) 041702. https://doi.org/10.1063/1.2191848 